\title{
Individual discrimination of freely swimming pulse-type electric fish from electrode array recordings
}

\author{
Paulo Matias $^{\mathrm{a}, *}$, Jan Frans Willem Slaets ${ }^{\mathrm{a}}$, Reynaldo Daniel Pinto ${ }^{\mathrm{a}}$ \\ ${ }^{a}$ Department of Physics and Interdisciplinary Science, São Carlos Institute of Physics, \\ University of São Paulo, Av. Trabalhador Sancarlense 400, \\ 13560-970 São Carlos, SP, Brazil
}

\begin{abstract}
Pulse-type weakly electric fishes communicate through electrical discharges with a stereotyped waveform, varying solely the interval between pulses according to the information being transmitted. This simple codification mechanism is similar to the one found in various known neuronal circuits, which renders these animals as good models for the study of natural communication systems, allowing experiments involving behavioral and neuroethological aspects. Performing analysis of data collected from more than one freely swimming fish is a challenge since the detected electric organ discharge (EOD) patterns are dependent on each animal's position and orientation relative to the electrodes. However, since each fish emits a characteristic EOD waveform, computational tools can be employed to match each EOD to the respective fish. In this paper we describe a computational method able to recognize fish EODs from dyads using normalized feature vectors obtained by applying Fourier and dual-tree complex wavelet packet transforms. We employ support vector machines as classifiers, and a continuity constraint algorithm allows us to solve issues caused by overlapping EODs and signal saturation. Extensive validation procedures with Gymnotus sp. showed that EODs can be assigned correctly to each fish with only two errors per million discharges.
\end{abstract}

Keywords: Neuroethology, Electric organ discharge, Classification, Dual-tree complex wavelet packet, Support vector machine, Continuity constraint

\section{Introduction}

Pulse-type weakly electric fishes such as Gymnotus sp. are known for emitting electric organ discharges (EODs) used for electrolocation and electrocommunication purposes [1, 2]. Because of the stereotyped nature of the electrical waveforms produced by these fish, research on electrocommunication typically focus on analyzing measurements derived only from the occurrence instant

\footnotetext{
*Corresponding author. Tel.: +55 16 33738090; fax: +55 1633739879 .

Email address: paulo.matias@usp.br (Paulo Matias)
} 
(timestamp) of EODs, for example its discrete difference - the inter-pulse interval (IPI) [3]. Few organisms allow the non-invasive examination of electrophysiological signals produced by a complex internal neuronal network as offered by this simple communication mechanism based on trains of electrical pulses.

Recording from freely swimming fish is challenging and traditional techniques based in arrays of electrodes fixed in the aquarium are still employed [6] 8]. The idea is similar to using an electrode array to record from neurons in the central nervous system of an animal [9, 10, however, here the spiking "neurons" are not stationary in space and the problem resembles, but it is even more complicated than, that of recording from arrays of electrodes which present drift along time [1]. Techniques allowing the precise detection and discrimination of EODs emitted by dyads or groups of freely moving fish with a minimum disturbance are invaluable tools for neuroethological research [4, 5, 12, 14, because they allow to study a plethora of social communication circumstances in a naturalistic setup. However, currently there is a lack of computational tools capable of accurately identifying the individual that emitted each pulse recorded during those experiments.

In principle, employing machine learning techniques to address the individual discrimination problem would be feasible since EOD waveforms vary from one fish to another [15]. But although the distinct waveforms of different individuals have been the object of study in reports on EOD variations related to geographical origin [16], characteristics of the waveforms have seldom been exploited for recognizing fish. There are also reports on changes of the waveform due to developmental transitions in juvenile fish [17], but the EOD of a certain individual does not change by factors other than fish movement [7, 18, during our experiment's time frame (a few hours).

Most of the existing works try to discriminate individuals by employing non-automatic procedures involving visual inspection of EOD pulse amplitude and duration [14, sometimes aided by video recordings that allow inferring fish position, which is then manually correlated to pulse amplitude and polarity changes [5]. Still, those methods are time-consuming and often applied solely to a few minutes of experimental data, and therefore they do not produce sufficient input to enable statistical and information-theoretic approaches [8] to study spike train coding [19].

An automated method was proposed [4 that first stored two template EODs, each one from a single fish, then computed the cross-correlation between every EOD acquired in a dyad experiment and each of the templates. Nevertheless, that approach had difficulties when EODs had similar pulse width or when both fish fired pulses almost at the same time.

In this paper we introduce a computational method able to discriminate fish within dyads surpassing the just mentioned limitations, given as input measurements from electrodes placed at fixed positions in an aquarium tank. Our method is essentially a two pass algorithm. First, signal portions strongly believed to contain an EOD emitted by a single fish are classified by a support vector machine [20], based on a normalized feature vector obtained by applying 


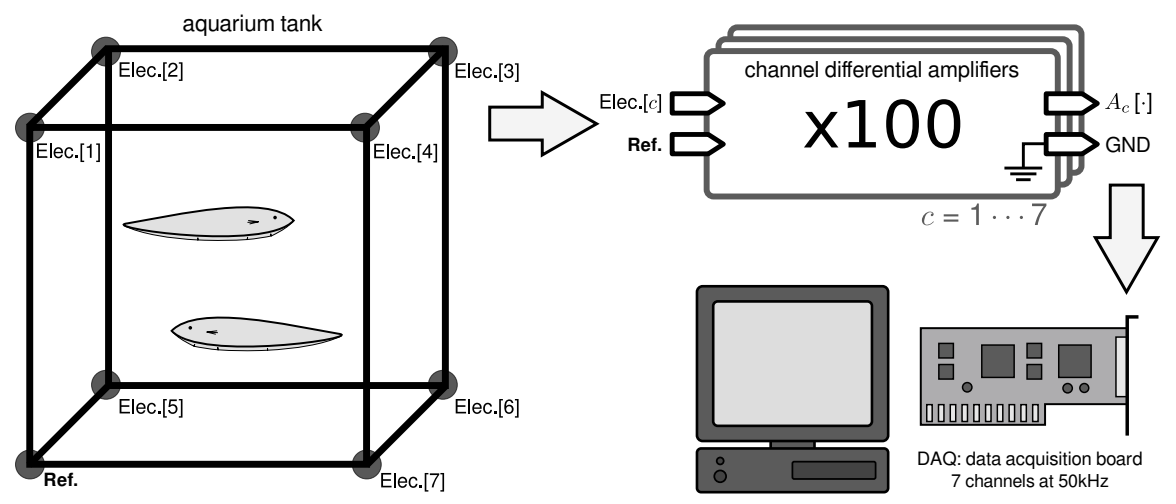

Figure 1: A pair of fish is placed in an aquarium tank containing eight electrodes in contact with water. One of the electrodes is chosen as a reference electrode (ref.), in respect to which the voltage of all other electrodes is differentially amplified with a 100 times gain. Signals sampled at $50 \mathrm{kHz}$ are then collected by a computer using a data acquisition board.

Fourier and dual-tree complex wavelet packet [21, 22, transforms. Then, the fact that waveforms vary continuously during fish movement is exploited to find EODs inside signal segments which might contain discharges from both fish.

We also perform an extensive validation procedure during which dipoles are attached directly to each fish to capture the EODs whose timestamps are then compared with the results of the developed algorithm in order to evaluate its error rate, which we estimate as being only two parts per million.

The paper is organized as follows. Section 2 introduces the experimental methods, explaining how measurements are carried, and also detailing the procedure we perform to validate our method and evaluate its accuracy. Section 3 describes both the discrimination method already present in literature and our proposed algorithm. Finally, results are presented in Section 4 and conclusions are drawn in Section 5 .

\section{Experimental methods}

\subsection{Experimental setup}

Our experimental setup is illustrated in Figure 1. Measurements are taken in a 64 liters glass aquarium tank containing eight stainless steel electrodes, located at the vertices of a $40 \mathrm{~cm}$ sided cube. The aquarium is mounted inside a Faraday cage to reduce the induction of external electrical noise. Electrodes with a diameter of $0.2 \mathrm{~mm}$ are inserted through the silicon glue at the corners of the aquarium, having about 1 to $2 \mathrm{~mm}$ of length in contact with the water. One of the electrodes is chosen as a reference, in respect to which the voltage of the other seven electrodes is differentially amplified 100 times using LM308 operational amplifiers. Once amplified, the seven signals are digitized at a sampling rate of 
$50 \mathrm{kHz}$ with a resolution of 12 bits by a National Instruments PCI MIO-16-E1 data acquisition board and stored in a personal computer.

When fish gets too close to the reference electrode, an exceeding voltage may be produced between the reference and other electrodes, saturating signals recorded from all of them at the same time. To avoid this issue, we fixed a piece of nylon tulle to the aquarium glass near the reference electrode, preventing fish from reaching it.

Our aquarium geometry and electrode placement is the same adopted in [8]. It is easy to replicate and provides an adequate signal to noise ratio (SNR) in at least one electrode independently of the fish position. However, nothing precludes the algorithm described in this paper from being used with other geometries, such as the round aquarium with multiple reference electrodes as described in [7].

\subsection{Experimental procedure}

Experiments with a fish dyad are comprised by two steps which can be carried in any desired order. We call one of these steps the training stage, which consists of placing apart in the aquarium each individual, in turn, for some minutes, during which the EODs of the freely swimming fish are collected. Typically, 15 minutes of acquisition are enough to collect on the order of $10^{5}$ EODs, sufficient for training and testing our classifier. In order to acquire good quality labeled training and testing data, covering the system dynamics over most of the operating range, it is important to get the fish to swim around all aquarium. In Gymnotus sp., this usually occurs naturally, as the fish has a tendency to explore the surroundings when it is moved to a different environment [8]. The experimenter can also arouse an inactive animal by mechanically disturbing the aquarium.

The other step, which we call the main experiment, consists of placing both fish at the same time inside the aquarium. Data acquired in this step can be discriminated by our algorithm, outputting a list of the occurrence instants of EODs emitted by each fish. These instants are the final product of our method, and can be analyzed and studied in order to research new behavior and codification schemes occurring in fish electrocommunication.

\subsection{Validation procedure}

To evaluate if our discrimination algorithm gave accurate results, we conducted experiments where electrodes were attached next to each fish and recorded in addition to the fixed electrode array already present in the aquarium. To keep the electrodes near the fish, the wires of the electrodes were intertwined to a nylon tulle, which was wrapped around the animals. Therefore, fish movement was fairly restrained during these tests, demanding that we manually moved the individuals around the aquarium, by pushing and pulling the wires, to simulate the position displacements of a freely swimming fish.

Instants of EOD emission could thus be directly obtained by applying a simple threshold to the signal captured from the electrode tied to each individual. 
These direct measurements were then compared with the output of our discrimination algorithm to which only the fixed electrode measurements were supplied as inputs.

Six different Gymnotus sp. dyads were used for carrying this validation procedure. For each dyad, two experiments were made. In the first one, both fish were restrained by nylon tulle cover and manually moved around the aquarium. Then, during the second experiment, one arbitrarily chosen individual of the dyad was freed and allowed to swim, while the other fish was kept enclosed near its electrode, and hence had its EOD instants directly measured.

\section{Discrimination methods}

\subsection{Cross-correlation method}

Before discussing our algorithm, we briefly introduce our implementation of the cross-correlation method already described in literature [4, which we will use for comparison purposes.

Given a signal $A[\cdot]$ containing an unlabeled EOD, the method computes the cross-correlation $T_{k} \star A$ for both $k=1$ and $k=2$. Each value of $k$ corresponds to one fish of the dyad, and $T_{k}$ is the signal template of such fish.

$$
\left(T_{k} \star A\right)[i]=\sum_{j=0}^{l_{w}-1} T_{k}[j] \cdot A[i+j]
$$

Where $l_{w}$ is the maximum length of an EOD in number of samples. The method then computes MaxCorr ${ }_{k}$, the maximum absolute value of the crosscorrelation for the $k$-th fish, considering all the possible EOD starting instants $i$ inside the signal $A[\cdot]$.

$$
\operatorname{MaxCorr}_{k}=\max _{i \in\left[-l_{w}, l_{w}\right]}\left|\left(T_{k} \star A\right)[i]\right|
$$

Then the $A[\cdot]$ signal is classified as containing an EOD from "fish 1" if $\left(\right.$ MaxCorr $_{1}-$ MaxCorr $\left._{2}\right) \geq$ DecThreshold. Otherwise, it is classified as "fish 2". The decision threshold (DecThreshold) did not exist in the original method. We introduced it as a parameter that can be varied to plot receiver operating characteristic (ROC) curves (e.g. Figure 5). Setting the threshold to zero recovers the results of the original method.

We choose templates $T_{k}$ by exhaustive search over all possible pairs of EODs extracted from subsets of $10^{3}$ labeled pulses selected at random from those collected during the training stage. We normalize them such that $\max _{j}\left|T_{k}[j]\right|=$ 1. We select the templates which provide the best classification accuracy on the other pulses of the training data, i.e. those not contained in the subsets where the template search is done.

An issue of the cross-correlation method is the low accuracy it presents when both fish of a dyad emit EODs of almost the same pulse duration. Also, the method does not include any means of dealing with overlapped EODs. Thus, 
our main challenges when implementing a new algorithm were to address these two points - classifying signals according to their fine structure even though they vary with fish position, and discriminating EODs even when both fish fire almost at the same time.

\subsection{Proposed algorithm}

Our discrimination algorithm works on signal segments which may contain either one or two EODs, respectively from a single fish or from both fish of a dyad. Subsection 3.2.1 describes how these segments are obtained from the acquired signals. Then, the algorithm conceptually consists of two main steps: the classification of single fish segments and the dissociation of signal segments containing EODs from both fish. The core of the first step is a support vector machine (SVM) classifier. The extraction and the selection of features which feed the SVM are detailed, respectively, in Subsections 3.2.2 and 3.2.3, and the application of the SVM to classify segments containing a single EOD is depicted in Subsection 3.2.4. The second step consists of applying a continuity constraint to allow the discrimination of two EODs present in the same segment, which is portrayed in Subsection 3.2.5.

\subsubsection{EOD segmentation}

Data both from the training stage and from the main experiment are segmented before analysis. The purpose of this procedure is to detect EODs, distinguishing them from background noise, and to estimate the time span of EOD activity on each detection.

Signals $A_{c}[\cdot]$ from all channels $c$ are passed by a low-pass finite impulse response filter (Hamming window, 11-tap, $2500 \mathrm{~Hz}$ cutoff), differentiated and squared. If the sum $I[\cdot]$ of the resulting signals surpasses a threshold value $t_{d}$, a segment of electric organ activity is detected.

$$
\begin{aligned}
F_{c}[\cdot] & =A_{c}[\cdot] * \text { Filter }[\cdot] \\
\Delta F_{c}[i] & =F_{c}[i+1]-F_{c}[i] \\
I[i] & =\sum_{c}\left(\Delta F_{c}[i]\right)^{2}
\end{aligned}
$$

Segment boundaries (starting and ending time instants $s$ and $e$ ) are determined by iterating over samples of the summed signal $I[\cdot]$, in both directions, starting at the position $I[d]$ where detection $\left(I[d]>t_{d}\right)$ took place, until a certain number $N_{b}$ of contiguous samples ahead of the boundary is found to be below a minimum value $t_{b}$, which is set to be lower than the detection threshold (such that $t_{b}<t_{d}$ ). This procedure is shown in Figure 2 and described by the equations below.

$$
\max \left\{\begin{aligned}
& s \in \mathbb{N}, s<d: \forall i \in \mathbb{N}, 1 \leq i \leq N_{b} \\
& \rightarrow I[s-i]<t_{b}
\end{aligned}\right\}
$$




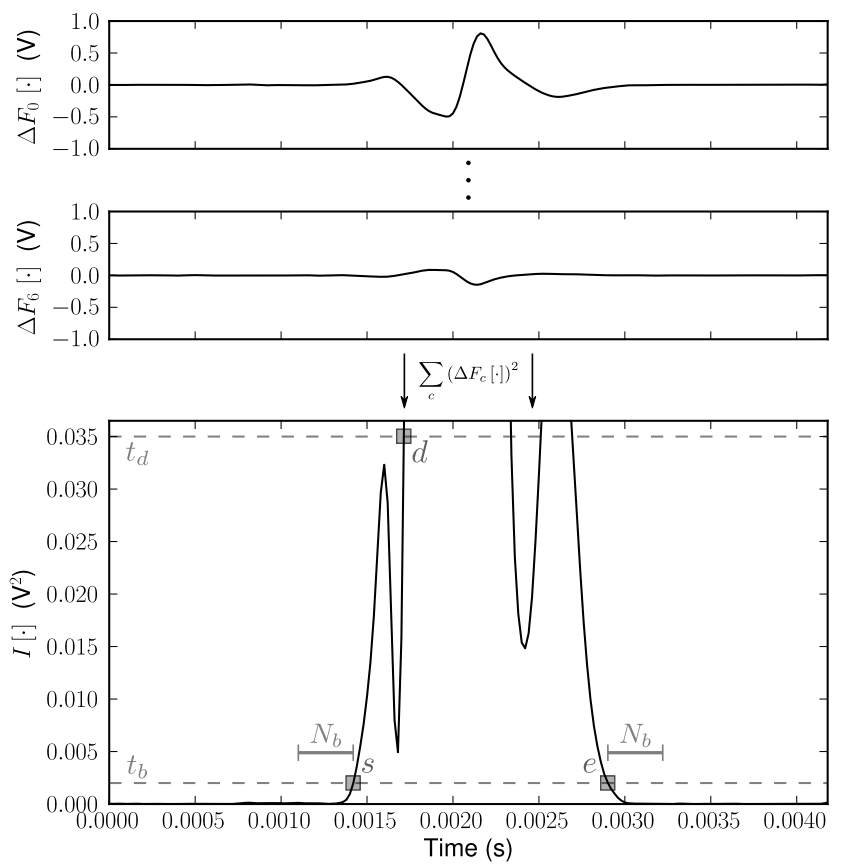

Figure 2: Signals $\Delta F_{c}[\cdot]$, obtained by filtering and differentiating experimental data collected from every channel $c$, are squared and summed to compute $I[\cdot]$. When $I[\cdot]$ surpasses a $t_{d}$ threshold, a segment of EOD activity is detected. To establish the $s$ (and $e$ ) segment boundaries, we look for a $N_{b}$ idle time occurring before (after) the signal goes below a $t_{b}$ threshold. 


$$
\min \left\{\begin{aligned}
& e \in \mathbb{N}, e>d: \forall i \in \mathbb{N}, 1 \leq i \leq N_{b} \\
& \rightarrow I[e+i]<t_{b}
\end{aligned}\right\}
$$

The parameter $t_{d}$ should be set just below the peak caused on $I[\cdot]$ by an EOD emitted when fish is at the position which leads to the minimum amplitude of acquired signals, i.e. at the middle point of the aquarium; $t_{b}$ should be adjusted above the maximum noise floor; and $N_{b}$ should be greater than the number of contiguous $I[\cdot]$ samples of an EOD that might be below $t_{b}$. We have developed a graphical user interface for adjusting these parameters which shows an interactive graphic similar to the one presented in the figure. For our experimental setup, we have chosen $t_{d}=0.06 \mathrm{~V}^{2}, t_{b}=0.0012 \mathrm{~V}^{2}$ and $N_{b}=16$.

The filtering operation carried before segmentation is meant to reduce the susceptibility to noise of $s$ and $e$ measurements. Filter choice is not critical as long as the spiking shape of the EOD is preserved. The purpose of the differentiation is to remove any DC component that might be left on the signal due to offsets in the acquisition system, avoiding spurious detections. Both operations are carried solely to aid EOD segmentation and their results are not used by the next steps of the algorithm. A less redundant approach is to replace these two operations by a signal reconstruction from the second leaf of the third level of the wavelet transform employed in Subsection 3.2.2 (equivalent to a sequence of low-pass, low-pass again, and finally high-pass filtering operations). This produces a frequency response which is very close to that of the already described $\Delta F_{c}[\cdot]$ signal, with the advantage of allowing the computed wavelet signal components to be subsequently reused.

In order to compute feature vectors from a segment containing a single EOD, a signal window of a fixed length is needed. We choose a length $l_{w}$ large enough to accommodate any single EOD, and center the segments into windows by computing window boundaries $s^{\prime}=\left(s+e-l_{w}\right) / 2$ and $e^{\prime}=s^{\prime}+l_{w}$ given the segment boundaries $s$ and $e$. In Gymnotus sp., pulse duration typically lies in the range of 1.8 to $2.2 \mathrm{~ms}$, thus we choose $l_{w}=128(2.56 \mathrm{~ms})$ when acquiring data at 50 $\mathrm{kHz}$.

\subsubsection{Feature extraction}

One set of features is independently computed for each channel $c$ of the original signal $A_{c}[\cdot]$, as it was before filtering and differentiation. We have selected feature extraction schemes which produce output less sensitive to fish position than the time-domain signals. A simple approach is to compute the Fourier transform $\tilde{A}_{c}[\cdot]=\mathfrak{F}\left\{A_{c}\left[s^{\prime} \cdots e^{\prime}\right]\right\}$ of pulse signal windows, take the complex magnitude $\left|\tilde{A}_{c}[\cdot]\right|$ of the resulting values — which represents the amplitude of each frequency component without phase information - and finally normalize the vector by dividing all the values by the largest one $\left(\left|\tilde{A}_{c}[\cdot]\right| / \max _{i}\left|\tilde{A}_{c}[i]\right|\right)$.

Nonetheless, it has been reported [23] that some species of fish are able to distinguish between pairs of different artificial EOD pulses possessing the same amplitudes of the frequency spectrum. In the cited work, the artificial EODs were constructed by a superposition of two time-shifted components. 
Therefore, the EODs differed only in the phase of the frequency spectrum and would be indistinguishable by the aforementioned means. We hypothesized that if fish can sense these variations in artificial EODs, real discharges could conceivably contain somewhat time-localized features, just like the time-shifted components of the artificial signal. For example, some range of frequencies could be particularly more prevalent at the beginning of the EODs emitted by a specific individual. This kind of information is useful for the classifier and can be extracted employing time-frequency techniques such as wavelet transforms.

Wavelet packet transforms build decomposition trees which, at each level, refine signal localization in frequency while loosing localization in time. We have chosen the dual-tree complex wavelet packet transform (DT-CWPT) because it is nearly shift-invariant, although it can sense time shifting when the decomposition level allows for sufficient time localization. The ordinary discrete wavelet packet transform (DWPT) does not hold this property and is susceptible to artifacts induced by phase variation at all levels of decomposition. This is an undesirable effect, since as far as possible we only want to take into account phase differences in EOD signals due to factors other than fish position.

We have implemented DT-CWPT using 20-tap Q-shift filters [24] and Daubechies wavelet filters with seven vanishing moments. The dual trees are combined to obtain a single tree comprising the complex magnitude of each component. As the transform is computed on windows of $l_{w}=128$ samples, trees decompose the signals into $\log _{2}\left(l_{w}\right)=7$ levels, each one containing 128 components. We independently normalize each level, by dividing all values by the largest one present in the same level of the tree.

In short, the DT-CWPT and Fourier transforms provide, respectively, $l_{w}$. $\log _{2}\left(l_{w}\right)=896$ and $l_{w} / 2=64$ signal components which, after normalized, can form the feature vectors.

\subsubsection{Feature selection}

The number of signal components provided by DT-CWPT and Fourier transforms is large (960 in total). If all these components were used as features, training times would be excessively long and the model would likely overfit. Therefore, we select only 20 signal components to constitute the feature vectors that feed the classifier.

To aid feature selection, we build two histograms for each signal component, one corresponding to each fish, as illustrated in Figure 3 . These histograms have the same bin intervals, so that the overlapping area, highlighted in the figure, can easily be calculated by integrating over the least of two bin heights at each point. The minimum overlapping area (zero) would be obtained in the ideal situation where training set EODs could be perfectly classified using only this single feature. The maximum area (one) would be reached in the worst case, when probability distributions of the signal component are almost the same in both fish, meaning the component is worthless for classification if used alone.

Although signal components cannot be considered independent from each other, we assume for simplicity that selecting the ones presenting the least histogram overlapping area makes a good feature set. For the problem at hand, 


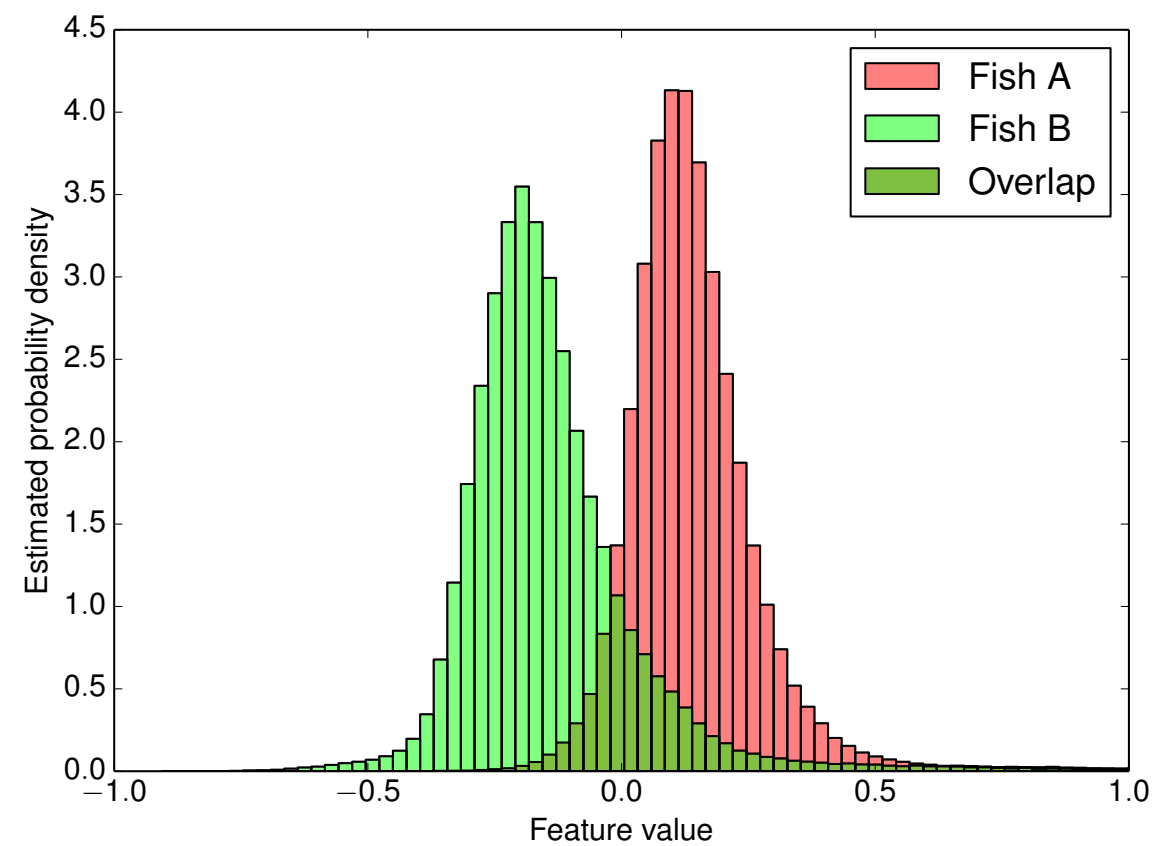

Figure 3: The histogram of a Fourier or wavelet component can be employed to estimate the probability distribution of its values. The overlapping area between histograms of the component in two distinct fish is used as a measure of the uncertainty of classifying EODs if only this single feature was known. We adopt the straightforward feature selection approach of choosing the components presenting the least histogram overlapping area. 
we verified empirically that this simple form of filter approach [25] for feature selection works well.

\subsubsection{Supervised classifier}

SVM classifiers were adopted because they frequently produce good results for a variety of problems [26]. Furthermore, mature and optimized SVM software libraries are readily available [27]. Each feature supplied to the classifier is rescaled to the $[-1,1]$ interval, in order to prevent dominance of the features spanning the larger numeric ranges. Our implementation uses by default the gaussian radial basis function (RBF) kernel, usually considered a good first choice, since depending on its parameters it can also behave like linear or sigmoid kernels [28].

Well-formed EOD signals (i.e. those which are non-saturated and have a good SNR, as verified by checking the EOD amplitude) obtained from a single fish during training stage are randomly distributed into three sets, each one containing on the order of $10^{4}$ EODs if training stage was carried for about 15 minutes per fish. One of them, the training set, is used to train the SVM model; another, the validation set, to count the number of errors throughout a grid search intended to find optimal RBF kernel and soft-margin parameters $(\gamma$ and $C$ ); and the third, the testing set, to estimate final SVM performance on single fish discharge classification.

Once the SVM model is trained, data collected from the main experiment can be processed. Signal segments obtained from this data may contain either an EOD emitted by a single fish or EODs fired by both fish almost at the same time. Diverse criteria are checked to establish with a high true negative rate (specificity) whether a certain segment contains a single EOD.

First, we dismiss segments whose length $(e-s)$ is greater than $\max \left\{\overline{l_{1}}+\right.$ $\left.\sigma_{l_{1}}, \overline{l_{2}}+\sigma_{l_{2}}\right\}$, where $\overline{l_{j}}$ is the mean and $\sigma_{l_{j}}$ is the standard deviation of the length of signal segments present in training data collected from the $j$-th fish alone. We also reject segments where less than $N_{r}$ of the available channels captured wellformed (good SNR, non-saturated) signals, and only consider pairs of adjacent segments such that the time interval between their starting instants is less than the minimum discharge period attainable by a single fish, in which case the EODs were probably emitted by different individuals.

Finally, the SVM model is employed to compute Platt [29, 30] probability estimators $p_{c}$ for every channel $c$ containing a well-formed signal during each segment. If one of the products $\prod_{c} p_{c}$ or $\prod_{c}\left(1-p_{c}\right)$ is above a certain threshold $t_{p}$, the associated segment is marked as being emitted by the first or by the second fish, respectively. Segments pertaining to an adjacent pair must have marks corresponding to different fish, otherwise the classification is deemed as incorrect.

We typically adopt $N_{r}=2$ and $t_{p}=0.95$. The larger the values of $N_{r}$ and $t_{p}$, the greater the overall specificity of the single fish classifier. We do not recommend choosing $N_{r}>2$ because it is rare to observe a well-formed EOD in more than two channels at the same time. Choosing $N_{r}=1$ can be effective if one fish stayed for several seconds of the experiment in a position 


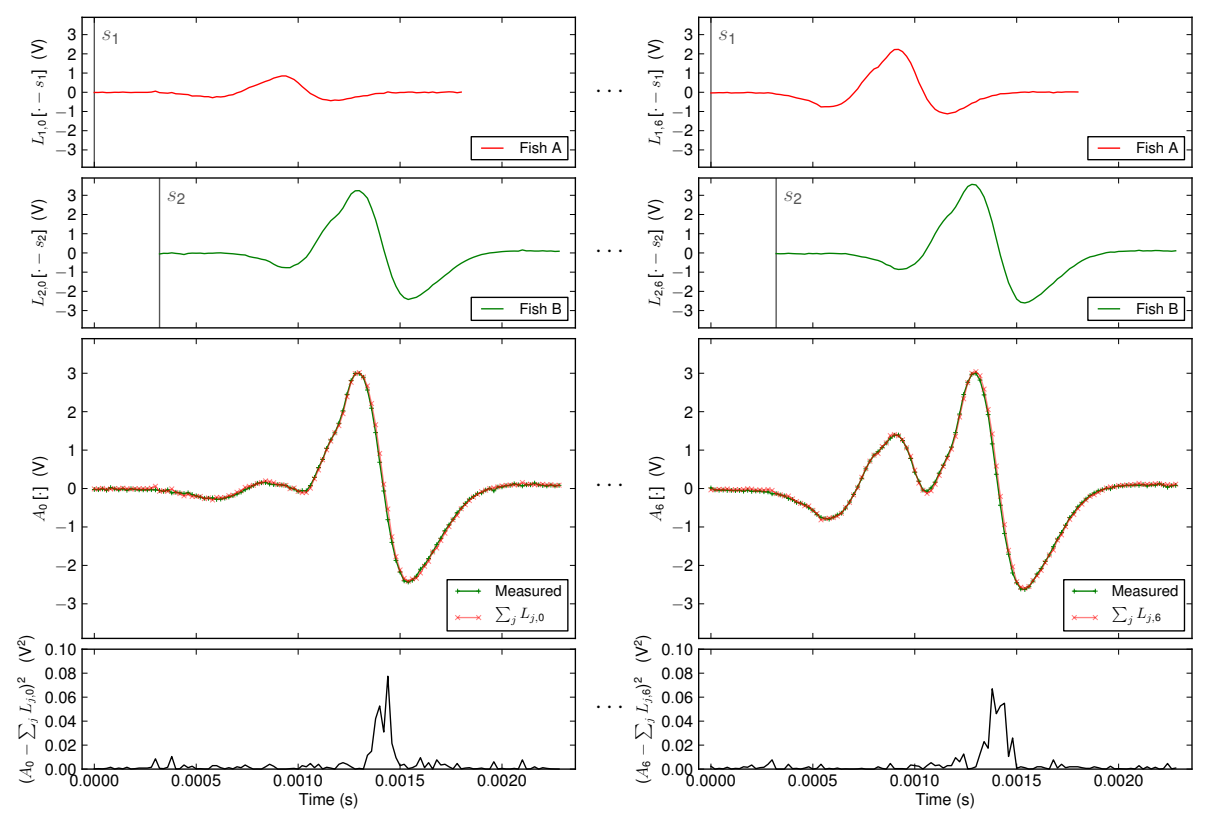

Figure 4: A segment of the $A_{c}[\cdot]$ signals may contain EODs emitted by both fish almost at the same time. In order to identify the positions $s_{j}$ where EODs emitted by the $j$-th fish start within the segment, we exploit the continuity property of EOD waveforms emitted by each fish. The figure is organized as a grid, where each column comprises graphics presenting data related to a certain channel $c$ of the signal. The first two lines show the $L_{j, c}$ vectors, which contain the last EOD previously recognized as being emitted by the $j$-th fish, displaced to the positions $s_{j}$ being evaluated. In the third line, both displaced vectors are summed, and the resulting $\sum_{j} L_{j, c}$ are compared to the measured $A_{c}$ signals. In this example, no saturation occurs, thus there is no need to apply the $\Xi$ function defined in the text. The instants $s_{j}$ are chosen to minimize the sum of all squared distances displayed in the fourth line of the figure.

of the aquarium where a well-formed EOD is captured by a single channel, but in these cases we recommend to compensate specificity by increasing $t_{p}$ to values in the order of 0.99. Classification errors on this step of the algorithm will propagate to the next pass (Subsection 3.2.5), therefore forcing a high specificity is important to get a final discrimination error in the order of parts per million even though the classification error of a single channel can reach hundreds of parts per million (see Table 1 .

\subsubsection{Continuity constraint}

Whenever an adjacent pair of segments fulfilling the aforementioned criteria is detected, for both $j \in\{1,2\}$, vectors $L_{j, c}[\cdot]$ are initialized with signals from every channel $c$, well-formed or not, belonging to the segment classified as containing a single EOD emitted by the $j$-th fish. Then, a continuity constraint is imposed to allow recognizing EODs present in subsequent signal segments, until the next criteria-satisfying adjacent pair is found. We assume that EOD waveforms collected from an individual vary smoothly, because fish position in 


\begin{tabular}{|c|c|}
\hline Dyad & Cross-validation accuracy \\
\hline \hline 1 & $99.9828 \%$ \\
\hline 2 & $99.9998 \%$ \\
\hline 3 & $99.9366 \%$ \\
\hline 4 & $99.9993 \%$ \\
\hline 5 & $99.9064 \%$ \\
\hline 6 & $99.9993 \%$ \\
\hline
\end{tabular}

Table 1: Results of a 10-fold cross-validation test carried applying our SVM based method on the entire data set collected during the training stage, for six different dyads.

the aquarium is a continuous function of time. This assumption is similar to an established approach [11] in spike sorting literature for solving the electrode drift problem.

Dissociation of signal segments containing EODs from both fish is thus formulated as an Euclidean distance minimization problem. Starting time instants $s_{j}$ of EODs emitted by the $j$-th fish are found by shifting the $L_{j, c}[\cdot]$ vectors to the positions $s_{j}$ being tested, summing shifted vectors corresponding to different fish, and comparing the resulting signal to the current segment $A_{c}[s \cdots e]$, as defined by the following equation and illustrated in Figure 4

$$
\underset{s_{1}, s_{2}}{\operatorname{argmin}} \sum_{i, c}\left(A_{c}[i]-\Xi\left(L_{1, c}\left[i-s_{1}\right]+L_{2, c}\left[i-s_{2}\right]\right)\right)^{2}
$$

In our notation, $L_{j, c}[i]$ evaluates to zero whenever $i$ is outside of the bounds $\left(0 \leq i<l_{w}\right)$. The function $\Xi(V[\cdot])$ roughly models the effect of differential amplifier output signal saturation. If the maximum and minimum output voltage swing values are given by $v_{s h}$ and $v_{s l}$, then $\Xi$ can be defined as follows.

$$
\Xi(V[k])= \begin{cases}v_{s h}, & \text { if } V[k] \geq v_{s h} \\ v_{s l}, & \text { if } V[k] \leq v_{s l} \\ V[k], & \text { otherwise }\end{cases}
$$

When testing different $s_{j}$ values, we allow $s_{1}$ to take a single value where $L_{1, c}\left[i-s_{1}\right]$ always evaluates to zero, meaning $L_{2, c}[\cdot]$ alone is compared to $A_{c}[s \cdots e]$, and vice versa. This way, the Euclidean distance minimization step can also be employed to detect signal segments containing a single EOD which were dismissed by the previous criteria due to its high specificity. When these segments are identified, the algorithm updates the $L_{j, c}[\cdot]$ vectors corresponding to the recognized fish $j$, in order to reflect the latest waveforms.

\section{Results}

First, we evaluated the SVM model alone regarding its ability to classify signal windows containing a single EOD. We present in Table 1 the results of a standard 10-fold cross-validation test conducted over all of the data collected 

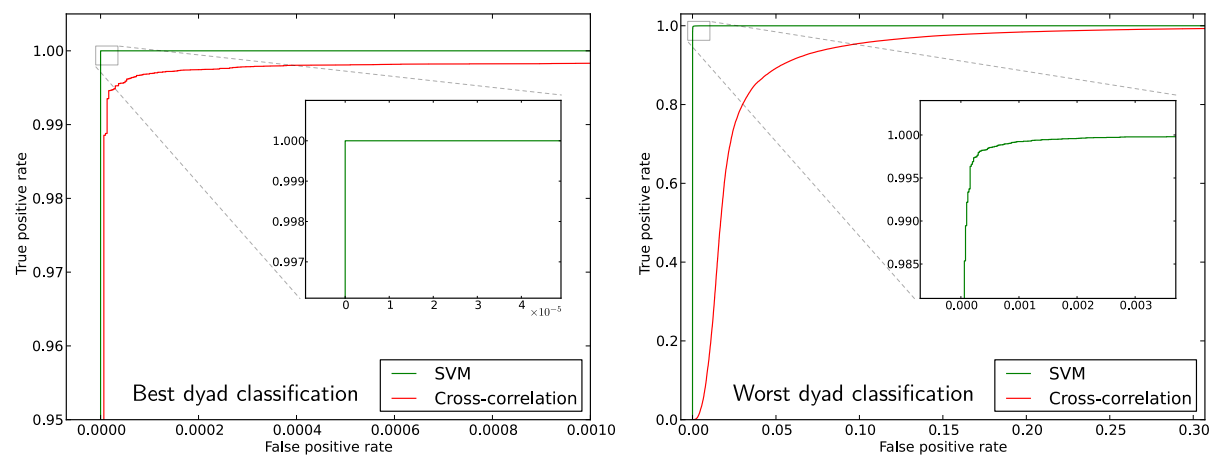

Figure 5: Receiver operating characteristic (ROC) curves comparing SVM and crosscorrelation methods for classifying signal windows of the testing set. Each window contained a single EOD emitted by an individual of a dyad (A and B fish). True positive rate denotes the ratio of EODs emitted by fish $\mathrm{A}$ which were correctly identified. Likewise, false positive rate corresponds to the ratio of incorrectly identified EODs emitted by fish B. Graphics were plotted for two different dyads, the ones which gave best and worst classification accuracy, respectively, among the six dyads used during experiments.

during the training stage. In other words, not taking into account the division into training, validation and testing sets mentioned in Subsection 3.2 .4

We also compared our SVM approach to the cross-correlation method described in Subsection 3.1, by plotting the receiver operating characteristic (ROC) curves shown in Figure 5 The data set used to obtain these curves is disjoint from the ones adopted to train the classifiers, i.e. curves were constructed based on classification results of the testing set, employing models trained only with the training set and with hyper-parameters optimized using the validation set. We plotted curves both for the best and for the worst case, corresponding to the dyads numbered 2 and 5 in Table 1, respectively. ROC results show that our SVM approach performs consistently better than the cross-correlation method. In the worst case data set, where SVM displayed particularly superior accuracy compared to cross-correlation, EODs emitted by different individuals had almost the same pulse duration, a situation on which the cross-correlation method is known [4] to work poorly.

Next, we analyzed data collected during the validation procedure (Subsection 2.3. By comparing direct electrode measurements with the discrimination algorithm results, we found a single non-detected EOD among $7.4 \times 10^{5}$ pulses, resulting in an error rate in the order of two parts per million EODs. For the discharge rate of a typical Gymnotus sp., this represents a mean interval of one to two hours of data collection between errors.

Finally, we observed inter-pulse interval (IPI) graphics plotted using results of the discrimination algorithm when applied to data collected with both fish of a dyad freely swimming, absent of any directly attached electrodes. As individuals were not restrained in any way during this test, it was conducted the closer way possible to a natural setting. Even though direct measurements are not available in this sort of experiment, a kind of validation can still be carried, because the 

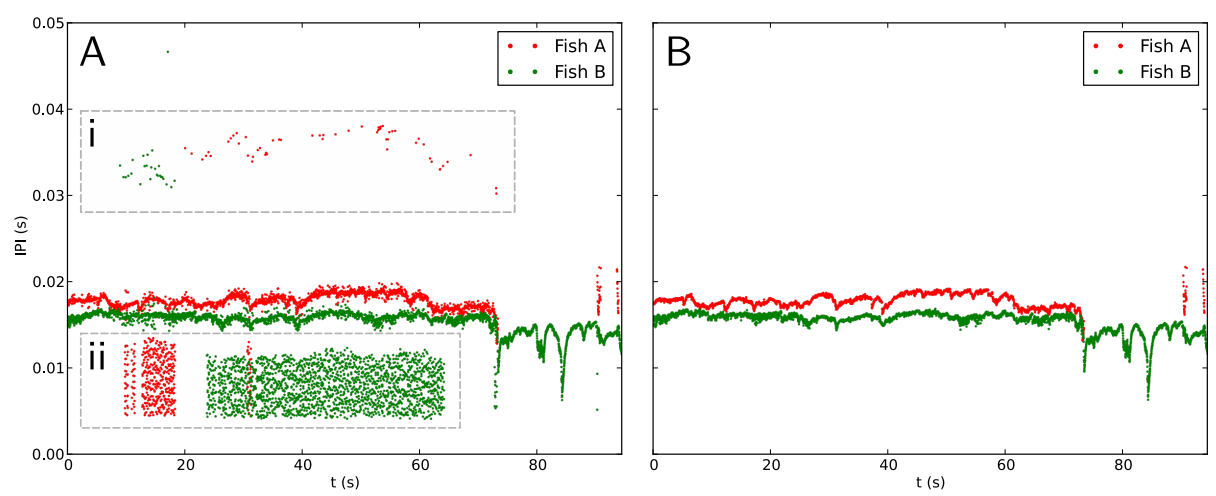

Figure 6: Inter-pulse interval (IPI) graphics plotted using two different discrimination algorithms, given the same data collected from a freely swimming fish dyad. On correct discrimination, IPI is expected to be a piecewise continuous function. Signals from which we computed IPI were not used during training. Sub-figure (A) shows results from a previous version of our algorithm which used the SVM alone (without applying the waveform continuity constraint), and therefore had difficulties when both fish emitted EODs almost at the same time or when some channel saturated. Discrimination errors can be easily pinpointed: region (i) contains missed EODs, and is located approximately at the double IPI of the baseline; region (ii) contains false positives, presenting IPIs below the baseline. Sub-figure (B) displays results from our fully implemented algorithm, as described in this paper. No errors can be pinpointed in its IPI graphic.

IPI curve for a fish is expected to be piecewise continuous. An individual can stop emitting EODs for a short period of time, but when it is emitting EODs, the pulse rate (and thus the IPI) varies smoothly.

The left panel of Figure 6 shows the IPI obtained employing a previous version of our algorithm, which did not incorporate the waveform continuity constraint discussed in Subsection 3.2.5. It simply classified signal windows using SVM and treated a window as containing pulses from both fish whenever signals of distinct channels were recognized as emitted by different fish. This led to a numerous amount of false positives when signal saturation occurred, and to some missed EODs when both fish fired almost at the same time. We show these results solely to illustrate that discrimination errors can be easily spotted in an IPI plot. One missed EOD appears as a point located at the double IPI of the baseline, as portrayed in the region (i) of the figure. Similarly, any EODs detected by the algorithm which did not really exist appear below the baseline, as displayed in region (ii).

On the other hand, the right panel of the figure shows the results of the fully implemented method, as described in Subsection 3.2. No errors can be pinpointed in this plot, as the IPI varies piecewise continuously for each fish.

However, we stress the fact that this IPI continuity property is not exploited by the discrimination algorithm. The continuity constraint used by our algorithm pertains only to the EOD waveform, which is a function of the spatial location of the fish, and is in no way related to the pulse rate. Therefore, no cyclic argument exists, and the fact that we observe smooth IPI curves when 
plotting the data obtained with our algorithm is one more reason to trust its correctness.

\section{Conclusions}

We have presented a method able to accurately recognize the individual which emitted each electric organ discharge (EOD) during experiments conducted with freely swimming Gymnotus sp. dyads. The obtained data is useful for analyzing and studying communication protocols employed by the animals in a range of interesting situations, like mating, dominance relation establishment and territorial dispute, besides being important for shedding more light on fundamental issues, such as efficiency and redundancy of communication signal coding, jamming avoidance response [31] and communication channel multiplexing mechanisms which might be present in these animals.

Our method requires only a simple experimental setup, consisting of an array of fixed electrodes, conventional operational amplifier circuits for conditioning signals, and a data acquisition system. Electrodes can be affixed to an aquarium or be mounted onto a structure which can be installed inside a fishpond. Unlike procedures carried in previous studies, no cameras are needed, allowing experiments to be easily carried in turbid waters, which are a common habitat of these animals [32. Also, we are able to reliably process a large amount of collected data, which is essential for the attainment of more faithful results when applying information theoretic and statistical approaches to analyze communication signals.

Experiments carried out with Gymnotus sp. gave outstanding discrimination results, therefore we believe this method could be applied to other species of pulse-type electric fish, although it remains to be attested if those species present individual distinguishable signatures which could be identified by our support vector machine (SVM) based classifier. Additionally, whilst we have devised and implemented the method only for dealing with a fish dyad, it can be naturally extended to process signals collected from more than two individuals. Notwithstanding, naively expanding the terms of the proposed continuity constraint for handling more individuals would be too much computationally expensive due to the processing power needed to deal with the large amounts of data. Therefore, some heuristics would need to be developed in order to reduce the optimization search space.

As a future work, we plan on implementing this algorithm in field-programmable gate array (FPGA) hardware, employing a shift-register-like architecture for the continuity constraint step. Such a device would allow us to obtain discriminated fish EOD instants with low latency and jitter characteristics. This would be suitable for conducting a new range of real-time closed-loop experiments such as involving a third artificial fish communicating with the dyad. 


\section{Acknowledgements}

This work was supported by a grant from the CAPES - Coordenação de Aperfeiçoamento de Pessoal de Nível Superior - Brazilian agency. We also acknowledge the FAPESP - Fundação de Amparo à Pesquisa do Estado de São Paulo - and CNPq - Conselho Nacional de Desenvolvimento Científico e Tecnológico - agencies for their financial support for past and future projects related to this work. We thank Lirio O. B. Almeida, Roland Köberle, Rafael T. Guariento and Krissia Zawadzki for reviewing our original manuscripts.

\section{References}

[1] M.V. Bennett, H. Grundfest, Electrophysiology of electric organ in Gymnotus carapo, J. Gen. Physiol. 42 (5) (1959) 1067-1104.

[2] A.A. Caputi, M.E. Castelló, P. Aguilera, O. Trujillo-Cenóz, Electrolocation and electrocommunication in pulse gymnotids: signal carriers, prereceptor mechanisms and the electrosensory mosaic, J. Physiol.-Paris 96 (5-6) (2002) 493-505.

[3] G.W. Max Westby, Has the latency dependent response of Gymnotus carapo to discharge-triggered stimuli a bearing on electric fish communication?, J. Comp. Physiol. 96 (4) (1975) 307-341.

[4] R.Y. Wong, C.D. Hopkins, Electrical and behavioral courtship displays in the mormyrid fish Brienomyrus brachyistius, J. Exp. Biol. 210 (2007) $2244-2252$.

[5] K. Gebhardt, M. Böhme, G. von der Emde, Electrocommunication behaviour during social interactions in two species of pulse-type weakly electric fishes (Mormyridae), J. Fish Biol. 81 (7) (2012) 2235-2254.

[6] G.W.M. Westby, Comparative studies of the aggressive behaviour of two gymnotid electric fish (Gymnotus carapo and Hypopomus artedi), Anim. Behav. 23 (1975) 192-213.

[7] J.J. Jun, A. Longtin, L. Maler, Precision measurement of electric organ discharge timing from freely moving weakly electric fish, J. Neurophysiol. 107 (7) (2012) 1996-2007.

[8] C.G. Forlim, R.D. Pinto, Automatic realistic real time stimulation/recording in weakly electric fish: Long time behavior characterization in freely swimming fish and stimuli discrimination, PLoS ONE 9 (1) (2014) e84885.

[9] G.W. Gross, E. Rieske, G.W. Kreutzberg, A. Meyer, A new fixed-array multi-microelectrode system designed for long-term monitoring of extracellular single unit neuronal activity in vitro, Neurosci. Lett. 6 (2-3) (1977) 101-105. 
[10] D.R. Kipke, R.J. Vetter, J.C. Williams, J.F. Hetke, Silicon-substrate intracortical microelectrode arrays for long-term recording of neuronal spike activity in cerebral cortex, IEEE T. Neur. Sys. Reh. 11 (2) (2003) 151-155.

[11] R.K. Snider, A.B. Bonds, Classification of non-stationary neural signals, J. Neurosci. Meth. 84 (1-2) (1998) 155-166.

[12] G. Batista, L. Zubizarreta, R. Perrone, A. Silva, Non-sex-biased dominance in a sexually monomorphic electric fish: Fight structure and submissive electric signalling, Ethology 118 (4) (2012) 398-410.

[13] R. Perrone, O. Macadar, A. Silva, Social electric signals in freely moving dyads of Brachyhypopomus pinnicaudatus, J. Comp. Physiol. A 195 (5) (2009) 501-514.

[14] M.E. Arnegard, B.A. Carlson, Electric organ discharge patterns during group hunting by a mormyrid fish, P. Roy. Soc. B-Biol. Sci. 272 (1570) (2005) 1305-1314.

[15] P.K. McGregor, G.W.M. Westby, Discrimination of individually characteristic electric organ discharges by a weakly electric fish, Anim. Behav. 43 (6) (1992) 977-986.

[16] J.R. Gallant, M.E. Arnegard, J.P. Sullivan, B.A. Carlson, C.D. Hopkins, Signal variation and its morphological correlates in Paramormyrops kingsleyae provide insight into the evolution of electrogenic signal diversity in mormyrid electric fish, J. Comp. Physiol. A 197 (8) (2011) 799-817.

[17] W.G.R. Crampton, C.D. Hopkins, Nesting and paternal care in the weakly electric fish Gymnotus (Gymnotiformes: Gymnotidae) with descriptions of larval and adult electric organ discharges of two species, Copeia 2005 (1) (2005) 48-60.

[18] C.D. Hopkins, Temporal structure of non-propagated electric communication signals, Brain Behav. Evol. 28 (1-3) (1986) 43-59.

[19] S.P. Strong, R. Koberle, R.R. de Ruyter van Steveninck, W. Bialek, Entropy and information in neural spike trains, Phys. Rev. Lett. 80 (1998) 197-200.

[20] C. Cortes, V. Vapnik, Support-vector networks, Mach. Learn. 20 (3) (1995) 273-297.

[21] I. Bayram, I.W. Selesnick, On the dual-tree complex wavelet packet and M-band transforms, IEEE Trans. Sig. Proc. 56 (6) (2008) 2298-2310.

[22] T. Weickert, C. Benjaminsen, U. Kiencke, Analytic wavelet packets: combining the dual-tree approach with wavelet packets for signal analysis and filtering, IEEE Trans. Sig. Proc. 57 (2) (2009) 493-502. 
[23] S. Paintner, B. Kramer, Electrosensory basis for individual recognition in a weakly electric, mormyrid fish, Pollimyrus adspersus (Günther, 1866), Behav. Ecol. Sociobiol. 55 (2) (2003) 197-208.

[24] N. Kingsbury, Design of Q-shift complex wavelets for image processing using frequency domain energy minimization, in: IEEE Image Proc., volume 1, 2003, pp. 1013-1016. doi 10.1109/ICIP.2003.1247137.

[25] G.H. John, R. Kohavi, K. Pfleger, Irrelevant features and the subset selection problem, in: Proc. of the 11th International Conference on Machine Learning, New Brunswick, NJ, 1994, pp. 121-129.

[26] D. Meyer, F. Leisch, K. Hornik, The support vector machine under test, Neurocomputing 55 (1-2) (2003) 169-186.

[27] C.-C. Chang, C.-J. Lin, LIBSVM: A library for support vector machines, ACM Trans. Intell. Syst. Technol. 2 (3) (2011) 27:1-27:27.

[28] C.-W. Hsu, C.-C. Chang, C.-J. Lin, A practical guide to support vector classification, Technical Report, Department of Computer Science, National Taiwan University, 2010. URL: http://www.csie.ntu.edu.tw/ cjlin/ papers/guide/guide.pdf.

[29] J.C. Platt, Probabilistic outputs for support vector machines and comparisons to regularized likelihood methods, in: Advances in Large-Margin Classifiers, MIT Press, 1999, pp. 61-74.

[30] H.-T. Lin, C.-J. Lin, R. Weng, A note on Platt's probabilistic outputs for support vector machines, Mach. Learn. 68 (3) (2007) 267-276.

[31] A. Capurro, C.P. Malta, Noise autocorrelation and jamming avoidance performance in pulse type electric fish, B. Math. Biol. 66 (4) (2004) 885905.

[32] O. Baffa, S.L. Correa, Magnetic and electric characteristics of the electric fish Gymnotus carapo, Biophys. J. 63 (2) (1992) 591 - 593. 